\title{
Prerequisites from Volumes 1 and 2
}

In this section we state some results from Volumes 1 and 2 which we use in what follows. If we formulate Lemma 1.4 from Volume 1 , then it is named below also as Lemma 1.4.

Lemma INTR. (a) If $M, N \triangleleft G$, then the quotient group $G /(M \cap N)$ is isomorphic to a subgroup of $G / M \times G / N$.

(b) If $Z$ is a cyclic subgroup of maximal order in an abelian p-group, then $Z$ is a direct factor of $G$. In particular, an abelian p-group is a direct product of cyclic subgroups.

(c) (Fitting's lemma) If $M$ and $N$ are nilpotent normal subgroups of a group $G$, then $\operatorname{cl}(M N) \leq \operatorname{cl}(M)+\operatorname{cl}(N)$.

(d) If $G$ is a p-group, then $G / \Phi(G)$ is elementary abelian of order, say d. Every minimal set of generators of $G$ contains exactly $d$ members.

(e) If $G$ is a p-group, then $\Phi(G)=G^{\prime} \mho_{1}(G)$.

Lemma 1.1. If $A$ is an abelian subgroup of index $p$ in a nonabelian $p$-group $G$, then $|G|=p\left|G^{\prime}\right||\mathrm{Z}(G)|$.

Theorem 1.2. Suppose that a nonabelian p-group has a cyclic subgroup of index $p$. Then one of the following holds:

(a) $G=\left\langle a, b \mid a^{2^{n-1}}=b^{2}=1, a^{b}=a^{-1}\right\rangle \cong \mathrm{D}_{2^{n}}$ is of class $n-1$, all elements of the set $G-\langle a\rangle$ are involutions.

(b) $G=\left\langle a, b \mid a^{2^{n-1}}=1, a^{2^{n-2}}=b^{2}, a^{b}=a^{-1}\right\rangle \cong \mathrm{Q}_{2^{\mathrm{n}}}$ is of class $n-1$, all elements of the set $G-\langle a\rangle$ have the same order 4 .

(c) $G=\left\langle a, b \mid a^{2^{n-1}}=1, a^{2^{n-2}}=b^{2}, a^{b}=a^{-1}\right\rangle \cong \mathrm{SD}_{2^{n}}$ is of class $n-1$, $\Gamma_{1}=\left\{D \cong \mathrm{D}_{2^{n-1}}, Q \cong \mathrm{Q}_{2^{n-1}},\langle a\rangle \cong \mathrm{C}_{2^{n-1}}\right\}, \Omega_{1}(G)=D, \Omega_{2}^{*}(G)=Q$.

(d) $G=\left\langle a, b \mid a^{p^{n-1}}=b^{p}=1, a^{b}=a^{1+p^{n-2}}\right\rangle \cong \mathrm{M}_{p^{n}}$ is of class 2 , where $n>3$ if $p=2, \mathrm{Z}(G)=\left\langle a^{p}\right\rangle, \Omega_{1}(G)=\left\langle a^{p^{n-2}}, b\right\rangle \cong \mathrm{E}_{p^{2}}$.

The groups $\mathrm{D}_{2^{n}}, \mathrm{Q}_{2^{n}}, \mathrm{SD}_{2^{n}}$ are called dihedral, generalized quaternion, semidihedral, respectively.

Proposition 1.3. If a p-group has only one subgroup of order $p$, then it is either cyclic or a generalized quaternion group. 
Lemma 1.4. Let $N$ be a normal subgroup of a $p$-group $G$. If $N$ has no $G$-invariant abelian subgroup of type $(p, p)$, then it is either cyclic or a 2-group of maximal class.

It follows from Lemma 1.4 that if $\mathrm{Z}_{2}(G)$ is cyclic, then $G$ is either cyclic or a 2-group of maximal class.

Lemma 1.6 (Taussky). If a nonabelian 2-group $G$ satisfies $\left|G: G^{\prime}\right|=4$, then it is a 2-group of maximal class.

Exercise 1.6(a). The number of abelian maximal subgroups in a nonabelian $p$-group $G$ is either 0 or 1 or $p+1$.

Exercise P1. If a nonabelian $p$-group $G$ has two distinct abelian maximal subgroups, then $\left|G^{\prime}\right|=p$.

Proposition 1.8 (Suzuki). If a nonabelian p-group $G$ has a self-centralizing abelian subgroup of order $p^{2}$, then $G$ is of maximal class. (For the converse assertion, see Theorem 9.6(c).)

Exercise 1.8(a). If a $p$-group $G$ is minimal nonabelian, then

$$
\left|G^{\prime}\right|=p, \quad \mathrm{~d}(G)=2, \quad|G: \mathrm{Z}(G)|=p^{2},
$$

and one of the following holds:

(a) $G=\left\langle a, b \mid a^{p^{m}}=b^{p^{n}}=1, a^{b}=a^{1+p^{m-1}}\right\rangle$ is metacyclic.

(b) $G=\left\langle a, b \mid a^{p^{m}}=b^{p^{n}}=1,[a, b]=c, c^{p}=1,[a, c]=[b, c]=1\right\rangle$ is nonmetacyclic. In that case, $\mho_{1}(G)=\left\langle a^{p}\right\rangle \times\left\langle b^{p}\right\rangle, G / \mho_{1}(G)$ is nonabelian of order $p^{3}$ and exponent $p$ unless $p=2$ (if $p=2$, then $G / \mho_{1}(G) \cong \mathrm{E}_{4}$ ).

(c) $G \cong \mathrm{Q}_{8}$.

Next, a group $G$ is nonmetacyclic if and only if $G^{\prime}$ is a maximal cyclic subgroup of $G$. The group $G$ is metacyclic if and only if $\left|\Omega_{1}(G)\right| \leq p^{2}$. No noncentral cyclic subgroup is normal in $G$.

Theorems 1.10 and 1.17. Suppose that a p-group $G$ is neither cyclic nor a 2-group of maximal class. Then

(a) $\mathrm{c}_{1}(G) \equiv 1+p\left(\bmod p^{2}\right)$.

(b) If $k>1$, then $\mathrm{c}_{k}(G) \equiv 0(\bmod p)$.

Theorem 1.20. If all subgroups of a nonabelian p-group $G$ are normal, then we have $G=Q \times E$, where $Q \cong \mathrm{Q}_{8}$ and $\exp (E) \leq 2$.

Exercise 1.69(a). Let $G$ be a $p$-group and let $H \neq K$ be two distinct maximal subgroups of $G$. Then $\left|G^{\prime}: H^{\prime} K^{\prime}\right| \leq p$. In particular, if $G^{\prime}$ is cyclic, there is $A \in \Gamma_{1}$ such that $\left|G^{\prime}: A^{\prime}\right| \leq p$. 
Lemma 4.2. Let $G$ be a p-group with $\left|G^{\prime}\right|=p$. Then $G=\left(A_{1} * A_{2} * \cdots * A_{s}\right) \mathrm{Z}(G)$, the central product, where $A_{1}, \ldots, A_{s}$ are minimal nonabelian, so $G / \mathrm{Z}(G)$ is elementary abelian of even rank. In particular, if $G / G^{\prime}$ is elementary abelian, then we have $\left|A_{1}\right|=\cdots=\left|A_{s}\right|=p^{3}, E=A_{1} * \cdots * A_{s}$ is extraspecial and $G=E Z(G)$.

Lemma 4.3. Let $E$ be a subgroup of a p-group $G$ with $\left|E^{\prime}\right|=p$ and $\mathrm{Z}(E)=\Phi(E)$. If $[G, E]=E^{\prime}$, then $G=E * \mathrm{C}_{G}(E)$.

Theorem 5.2 (Hall's enumeration principle). Let $G$ be a p-group and $\mathcal{M}$ be a set of proper subgroups of $G$. Given $H \leq G$, let $\alpha(H)$ be the number of members of the set $\mathcal{M}$ contained in $H$. Then

$$
\alpha(G) \equiv \sum_{H \in \Gamma_{1}} \alpha(H) \quad(\bmod p) .
$$

Theorems 5.3 and 5.4. Suppose that a p-group $G$ of order $p^{m}$ is neither cyclic nor a 2-group of maximal class and $1 \leq n<m$. Then $\mathrm{s}_{n}(G) \equiv 1+p\left(\bmod p^{2}\right)$.

Theorem 5.8. Let $G$ be a group of order $p^{m}>p^{3}$ and exponent $p, 2<n<m$. Let $\mathcal{M}$ denote the set of all 2-generator subgroups of order $p^{n}$ in $G$ and $\alpha(K)$ the number of elements of the set $\mathcal{M}$ contained in $K \leq G$.

(a) If $n=m-1$, then $\alpha(G) \in\left\{0, p, p^{2}\right\}$.

(b) $p$ divides $\alpha(G)$.

Theorem 7.1. Let $G$ be a p-group.

(a) Regularity is inherited by sections.

(b) If $\operatorname{cl}(G)<p$ or $|G| \leq p^{p}$ or $\exp (G)=p$, then $G$ is regular.

(c) If $\mathrm{K}_{p-1}(G)$ is cyclic, then $G$ is regular.

Theorem 7.2. Suppose that $G$ is a regular p-group.

(b) $\exp \left(\Omega_{n}(G)\right) \leq p^{n}$.

(c) $\mho_{n}(G)=\left\{x^{p^{n}} \mid x \in G\right\}$.

(d) $\left|\Omega_{n}(G)\right|=\left|G: \mho_{n}(G)\right|$.

Theorem 9.5. Let $G$ be a group of maximal class and order $p^{m}, m \leq p+1$. Then $\Phi(G)$ and $G / \mathrm{Z}(G)$ have exponent $p$. If $m=p+1$, then $G$ is irregular and $\left|\mho_{1}(G)\right|=p$.

Theorem 9.6. Let $G$ be a group of maximal class and order $p^{m}, p>2, m>p+1$. Then $G$ is irregular and

(a) $\left|G: \mho_{1}(G)\right|=p^{p}$. In particular, $\mho_{1}(G)=\mathrm{K}_{p}(G)$.

(b) There is $G_{1} \in \Gamma_{1}$ such that $\left|G_{1}: \mho_{1}\left(G_{1}\right)\right|=p^{p-1}$. 
(c) $G$ has no normal subgroups of order $p^{p}$ and exponent $p$ (here the condition that $m>p+1$ is essential). Moreover, if $N \triangleleft G$ and $|G: N|>p$, then $N$ is absolutely regular since $N<G_{1}$. Next, if $N \triangleleft G$ of order $p^{p-1}$, then $\exp (N)=p$. In particular, $G$ has no normal cyclic subgroups of order $p^{2}$.

(d) Let $Z_{2}=Z_{2}(G)$ be a normal subgroup of order $p^{2}$ in $G, G_{0}=\mathrm{C}_{G}\left(Z_{2}\right)$. Then $G_{0}$ is regular such that $\left|\Omega_{1}\left(G_{0}\right)\right|=p^{p-1}$.

(e) Let $\Gamma_{1}=\left\{M_{1}=G_{0}, M_{2}, \ldots, M_{p+1}\right\}$, where $G_{0}$ is defined in (d). Then the subgroups $M_{2}, \ldots, M_{p+1}$ are of maximal class (and so irregular; see Theorem 9.5). Thus the subgroups $G_{1}$ from (b) and $G_{0}$ from (d) coincide. In what follows we call $G_{1}$ the fundamental subgroup of $G$.

(f) In this part, $m \geq 3$ (i.e., we do not assume as in other parts that $m>p+1$ ). The group $G$ has an element a such that $\left|\mathrm{C}_{G}(a)\right|=p^{2}$, i.e., $\mathrm{C}_{G}(a)=\left\langle a, \mathrm{~K}_{m-1}(G)\right\rangle$.

Theorem 9.7. A nonabelian p-group $G$ is of maximal class if and only if $G / \mathrm{K}_{p+1}(G)$ is of maximal class.

Theorem 9.8. (a) Absolutely regular p-groups $G$ (i.e., $G$ with $\left|G / \mho_{1}(G)\right|<p^{p}$ ) are regular.

(b) If a p-group $G$ is such that $\left|G^{\prime} / \mho_{1}\left(G^{\prime}\right)\right|<p^{p-1}$, then $G$ is regular.

(c) Any irregular p-group $G$ has a characteristic subgroup $R$ of order $\geq p^{p-1}$ and exponent $p$ such that $R \leq G^{\prime}$.

Theorem 9.11. A p-group $G, p>2$, is metacyclic if and only if $\left|G / \mho_{1}(G)\right| \leq p^{2}$.

Exercise 9.13. Let $G$ be a $p$-group of maximal class, $p>2$, and $H<G$. Then $\mathrm{d}(H) \leq p$. If $\mathrm{d}(H)=p$, then $G \cong \Sigma_{p^{2}} \in \operatorname{Syl}_{p}\left(\mathrm{~S}_{p^{2}}\right)$. In particular (Blackburn), if $G$ is a $p$-group of maximal class, $p>2, N \triangleleft G$ and $G / N \cong \Sigma_{p^{2}}$, then $N=\{1\}$.

Theorem 10.1. Let $p^{n}>2$ and let $A<G$ be abelian of exponent $p^{n}$. Then the number of abelian subgroups $B \leq G$ of order $p|A|$ that contain $A$ is congruent to 1 modulo $p$.

Corollary 10.2. Let $N$ be a normal subgroup of a p-group $G$ and let $A<N$ be a maximal $G$-invariant abelian subgroup of exponent $\leq p^{n}$, where $p^{n}>2$. Then we have $\Omega_{n}\left(\mathrm{C}_{N}(A)\right)=A$.

Theorems 10.4 and 10.5. Suppose that $G$ is a p-group, $p>2$. Let $\epsilon_{n}(G)$ be the number of elementary abelian subgroups of order $p^{n}$ in $G$. If $k \in\{3,4\}$ and $\epsilon_{k}(G)>0$, then we have $\epsilon_{k}(G) \equiv 1(\bmod p)$.

Exercise P2. Let $G$ and $k$ be such as in the previous theorem.

(a) If $\mathrm{Z}_{k}(G)$ has no $G$-invariant elementary abelian subgroup of order $p^{k}$, then we have $\epsilon_{k}(G)=0$.

(b) If a $G$-invariant subgroup $N$ of $G$ has no $G$-invariant elementary abelian subgroup of order $p^{k}$, then $\epsilon_{k}(N)=0$. 
Lemma 10.8. Suppose that $G$ is a minimal nonnilpotent group. Then $G=P Q$, where $P \in \operatorname{Syl}_{p}(G), Q=G^{\prime} \in \operatorname{Syl}_{q}(G)$ and

(a) $P$ is cyclic, $\mid P:(P \cap \mathrm{Z}(G) \mid=p$.

(b) $Q$ is either elementary abelian or special, $|Q / \Phi(Q)|=q^{b}$, where $b$ is the order of $q$ modulo $p$.

(c) If $Q$ is special, then $b$ is even and $\Phi(Q)=\mathrm{Z}(Q) \leq \mathrm{Z}(G),|\Phi(Q)| \leq p^{b / 2}$.

Theorem (Frobenius' normal p-complement theorem). If a group $G$ has no $p$-closed minimal nonnilpotent subgroup of order divisible by $p$, then $G$ has a normal p-complement $(=p$-nilpotent $)$.

Theorem (Burnside's normal p-complement theorem). If a Sylow p-subgroup of a group $G$ is contained in the center of its normalizer, then $G$ is p-nilpotent.

Proposition 10.17. If $B \leq G$ is a nonabelian subgroup of order $p^{3}$ in a $p$-group $G$ and $C_{G}(B)<B$, then $G$ is of maximal class.

Remark 10.5. Let $H<G$ and assume that $\mathrm{N}_{G}(H)$ is of maximal class. Then $G$ is also of maximal class.

Proposition 10.19. Suppose that $H$ is a nonabelian subgroup of order $p^{3}$ in a metacyclic p-group $G$. If $p>2$, then $G=H$. If $p=2$, then $G$ is of maximal class.

Proposition 10.28. A nonabelian p-group $G$ is generated by minimal nonabelian subgroups. In particular, if, in addition, $G$ is not minimal nonabelian, it contains two nonconjugate minimal nonabelian subgroups.

Theorem 10.33. If all minimal nonabelian subgroups of a nonabelian 2-group $G$ are generated by involutions, then $G=\langle x\rangle \cdot A$, where $A \in \Gamma_{1}$ is abelian and all elements in $G-A$ are involutions (such $G$ is said to be generalized dihedral).

Theorem 12.1. (a) If a p-group $G$ has no normal subgroup of order $p^{p}$ and exponent $p$, then $G$ is either absolutely regular or irregular of maximal class.

(b) If an irregular p-group $G$ has an absolutely regular maximal subgroup $H$, then it is either of maximal class or $G=H \Omega_{1}(G)$, where $\Omega_{1}(G)$ is of order $p^{p}$ (and, of course, of exponent $p$ ).

Exercise P3. If $\Omega_{p}(G)$ has no $G$-invariant subgroup of order $p^{p}$ and exponent $p$, then $G$ is either absolutely regular or of maximal class.

Theorem 12.12. Let a group $G$ of order $p^{m}$ be neither absolutely regular nor of maximal class and suppose that $H \in \Gamma_{1}$ is of maximal class. Then

(a) $\mathrm{d}(G)=3$.

(b) Set $v=m-2$ if $m \leq p+1$ and $v=p$ if $m>p+1$. Then $G / \mathrm{K}_{v}(G)$ is of order $p^{v+1}$ and, if $m>4$, it is of exponent $p$. 
(c) Exactly $p^{2}$ maximal subgroups of $G$ are of maximal class. If, in addition, $p>2$ and $m>4$, then the remaining $p+1$ maximal subgroups of $G$ have no two generators and their intersection $\eta(G)$ has index $p^{2}$ in $G$.

Theorem 13.2. Suppose that a p-group $G$ is neither absolutely regular nor of maximal class. Then

(a) $\mathrm{c}_{1}(G) \equiv 1+p+\cdots+p^{p-1}\left(\bmod p^{p}\right)$.

(b) If $k>1$, then $\mathrm{c}_{k}(G) \equiv 0\left(\bmod p^{p-1}\right)$.

Corollary 13.3. Suppose that an irregular p-group $G$ is neither absolutely regular nor of maximal class and $k<p$. Then it has a normal subgroup $M$ of order $p^{k}$ and exponent $p$, and the number of subgroups in $G$ of order $p^{k+1}$ and exponent $p$ containing $M$ is $\equiv 1(\bmod p)$.

Theorem 13.5. If a p-group $G$ is neither absolutely regular nor of maximal class, then $e_{p}(G) \equiv 1(\bmod p)$ (here $e_{n}(G)$ is the number of subgroups of order $p^{n}$ and exponent $p$ in $G)$.

Exercise P4. Let $N$ be a normal subgroup of a $p$-group $G$. If $N$ has no $G$-invariant subgroup of order $p^{p}$ and exponent $p$, then it is either absolutely regular or of maximal class.

Theorem 13.6. Let a group $G$ of order $p^{m}$ be neither absolutely regular nor of maximal class, let $n$ be a natural number with $m>n \geq p+1$. Denote by $\alpha(G)$ the number of subgroups of maximal class and order $p^{n}$ in $G$. Then $p^{2}$ divides $\alpha(G)$.

Theorem 13.7. Let $p>2$ and suppose that a p-group has no normal elementary abelian subgroup of order $p^{3}$. Then one of the following holds:

(a) $G$ is metacyclic.

(b) $G$ is a 3-group of maximal class not isomorphic to a Sylow 3-subgroup of the symmetric group of degree 9.

(c) $G=\Omega_{1}(G) C$, where $\left|\Omega_{1}(G)\right|=p^{3}$ and $C$ is cyclic.

Exercise P5. Let $G$ be a $p$-group, $p>2$, and suppose that $\Omega_{1}(G)$ has no $G$-invariant elementary abelian subgroup of order $p^{3}$. Then $G$ is one of the groups from Theorem 13.7.

Exercise 13.10(a). Let $H<G$, where $G$ is a $p$-group. If every subgroup of $G$ of order $p|H|$ containing $H$ is of maximal class, then $G$ is also of maximal class.

Proposition 13.18. Let $G$ be a p-group, and let $M<G$ be of maximal class.

(a) Set $D=\Phi(M), N=\mathrm{N}_{G}(M)$ and $C=\mathrm{C}_{N}(M / D)$. Let $t$ be the number of subgroups $K \leq G$ of maximal class such that $M<K$ and $|K: M|=p$. Then $t=\mathrm{c}_{1}(N / M)-\mathrm{c}_{1}(C / M)$. If $G$ is not of maximal class, then $t \equiv 0(\bmod p)$. 
(b) Suppose, in addition, that $M$ is irregular and $G$ is not of maximal class and a positive integer $k$ is fixed. Then the number $t$ of the subgroups $L<G$ of maximal class and order $p^{k}|M|$ such that $M<L$ is a multiple of $p$.

Theorem 36.1. Let $G$ be a nonabelian p-group and $R<G^{\prime}$ be a $G$-invariant subgroup of index $p$. Then $G$ is metacyclic if and only if $G / R$ is metacyclic.

Corollary 36.6 (Blackburn). Suppose that a nonabelian p-group $G$ and all its maximal subgroups are two-generator. Then $G$ is either metacyclic or $p>2$ and $\mathrm{K}_{3}(G)=$ $\mho_{1}(G)$ has index $p^{3}$ in $G$ (in the last case, $\left|G: G^{\prime}\right|=p^{2}$ ).

Theorem 36.16. Suppose that a p-group $G$ is such that $G / \mho^{2}(G)$ is of maximal class. Then $G$ is also of maximal class.

Exercise P6. If a $p$-group $G$ is such that $G / \mho_{2}(G)$ is of maximal class, then $G$ is also of maximal class.

Lemma 42.1. Let $G$ be a group of order $p^{m}$ satisfying $\left|\Omega_{2}(G)\right| \leq p^{p+1}<|G|$. Then one of the following holds:

(a) $G$ is an $\mathrm{L}_{p}$-group.

(b) $G$ is absolutely regular.

(c) $p=2, G$ is metacyclic and

$$
G=\left\langle a, b \mid a^{2^{m-2}}=b^{8}=1, a^{b}=a^{-1}, a^{2^{m-3}}=b^{4}, m>4\right\rangle .
$$

Here $\mathrm{Z}(G)=\left\langle b^{2}\right\rangle, G^{\prime}=\left\langle a^{2}\right\rangle$ and $G / G^{\prime}$ is abelian of type $(4,2), \Phi(G)=$ $\left\langle a^{2}, b^{2}\right\rangle, \Omega_{2}(G)=\left\langle a^{2^{m-4}}, b^{4}\right\rangle$.

Corollary 44.6. A p-group is metacyclic if and only if one of the following quotient groups is metacyclic:

$$
G / \Phi\left(G^{\prime}\right), \quad G / \mathrm{K}_{3}(G), \quad G / \mho_{1}\left(G^{\prime}\right), \quad \text { and, if } p>2 \text {, then } G / \mho_{1}(G) .
$$

Corollary 44.9. If $G / \mho_{2}(G)$ is a metacyclic 2-group, then $G$ is also metacyclic.

Theorem 44.12. Suppose that $N$ is a two-generator normal subgroup of a p-group $G$. If $N \leq \Phi(G)$, then $N$ is metacyclic.

Theorem 50.1. Let $G$ be a 2-group which has no normal elementary abelian subgroup of order 8 . Then $G$ has a normal metacyclic subgroup $N$ such that $G / N$ is isomorphic to a subgroup of $\mathrm{D}_{8}$.

Lemma 57.1. Let $G$ be a nonabelian $p$-group and let $A$ be a maximal abelian normal subgroup of $G$. Then for any $x \in G-A$, there is $a \in A$ such that $[a, x] \neq 1,[a, x]^{p}=1$, and $[a, x, x]=1$ which implies that $\langle a, x\rangle$ is minimal nonabelian. Therefore $G$ is generated by its minimal nonabelian subgroups.

In particular, if all minimal nonabelian subgroups of a nonabelian $p$-group $G, p>2$, have exponent $p$, then $\mathrm{H}_{p}(G)$ is abelian. (For a stronger result, see Mann's commentary to Problem 115.) 
Lemma 57.2. Let $G$ be a nonabelian 2-group all of whose minimal nonabelian subgroups are of exponent 4 and let $A$ be a maximal normal abelian subgroup of $G$. Then all elements in $G-A$ are of order $\leq 4$ and so either $\exp (A)=2$ or $\exp (A)=\exp (G)$. If $x \in G-A$ with $x^{2} \in A$, then $x$ inverts each element in $\mho_{1}(A)$ and in $A / \Omega_{1}(A)$. If $\exp (G)>4$, then either $G / A$ is cyclic of order $\leq 4$ or $G / A \cong \mathrm{Q}_{8}$.

Lemma 65.2(a). If $G$ is a nonabelian two-generator p-group and $G^{\prime} \leq \Omega_{1}(\mathrm{Z}(G))$, then $G$ is minimal nonabelian.

Theorems 66.1 and 69.1. If $G$ is a minimal nonmetacyclic p-group, then one of the following holds:

(a) $G$ is of order $p^{3}$ and exponent $p$.

(b) $G$ is a 3-group of maximal class and order $3^{4}$.

(c) $G \cong \mathrm{D}_{8} * \mathrm{C}_{4}=\mathrm{Q}_{8} * \mathrm{C}_{4}$ is of order 16 .

(d) $G=\mathrm{Q}_{8} \times \mathrm{C}_{2}$.

(e) $G$ is special of order $2^{5}$ with $|\mathrm{Z}(G)|=4$, exactly one maximal subgroup of $G$ is abelian.

It follows from the previous theorem that a $p$-group $G$ is metacyclic in any of the following cases: (i) every subgroup of $G$ of exponent $\leq p^{2}$ is metacyclic (in particular, if $\Omega_{2}(G)$ is metacyclic), (ii) every three-generator subgroup of $G$ is metacyclic.

Exercise P7. A $p$-group is metacyclic if one of the following holds:

(a) $\Omega_{2}(G)$ is metacyclic.

(b) $G / \mho_{2}(G)$ is metacyclic.

(c) $G / \mathrm{K}_{3}(G) \Phi\left(G^{\prime}\right)$ is metacyclic.

Exercise P8. If a 2-group $G$ and all its maximal subgroups are two-generator, then $G$ is metacyclic.

Propositions 71.3-71.5. If a two-generator p-group is a nonmetacyclic $\mathcal{A}_{2}$-group, then $p>2$.

Theorems 82.1-82.3. Let $G$ be a 2-group with exactly three involutions and assume that $\mathrm{Z}(G)$ is noncyclic. Then $G$ has a normal metacyclic subgroup $M$ such that $G / M$ is elementary abelian of order at most 4.

Theorem 90.1. Let $G$ be a nonabelian 2-group all of whose minimal nonabelian subgroups are isomorphic to $\mathrm{D}_{8}$ or $\mathrm{Q}_{8}$. Then $G$ is one of the following groups:

(a) $G$ is generalized dihedral (i.e., $\left|G: \mathrm{H}_{2}(G)\right|=2$ ).

(b) $G=H \mathrm{Z}(G)$, where $H$ is of maximal class and $\mho_{1}(\mathrm{Z}(G)) \leq \mathrm{Z}(H)$.

(c) $G=H \mathrm{Z}(G)$, where $H$ is extraspecial and $\mho_{1}(\mathrm{Z}(G)) \leq \mathrm{Z}(H)$. 
Corollary 90.2. Let $G$ be a nonabelian 2-group in which any two noncommuting elements generate a subgroup of maximal class. Then $G$ is one of the groups (a), (b) or (c) from Theorem 90.1. Conversely, each group in (a), (b) or (c) of Theorem 90.1 satisfies the assumption of our corollary.

Exercise P9. Let $H=\langle a, b\rangle$ be a two-generator $p$-group with $H^{\prime}$ of order $p$. Then $\Phi(H)=\left\langle a^{p}, b^{p},[a, b]\right\rangle$ and $H$ is minimal nonabelian.

Exercise P10. Let $G$ be a $p$-group with $\left|G^{\prime}\right|=p$. If $H$ is a minimal nonabelian subgroup of $G$, then $G=H C_{G}(H)$.

Exercise P11. All $p^{2}+p+1$ subgroups of order $p^{2}$ in an elementary abelian group $E=\langle a, b, c\rangle$ of order $p^{3}$ are: $\langle a, b\rangle,\left\langle a, b^{i} c\right\rangle(p+1$ subgroups containing $\langle a\rangle)$ and $\left\langle a^{j} b, a^{k} c\right\rangle$ ( $p^{2}$ subgroups not containing $\left.\langle a\rangle\right)$, where $i, j, k$ are any integers modulo $p$.

Corollary 92.7. Let $G$ be a non-Dedekindian p-group and let $R(G)$ be the intersection of all nonnormal subgroups. If $R(G)>\{1\}$, then $p=2,|R(G)|=2$ and $G$ is one of the following groups:

(a) $G \cong \mathrm{Q}_{8} \times \mathrm{C}_{4} \times \mathrm{E}_{2} s, s \geq 0$.

(b) $G \cong \mathrm{Q}_{8} \times \mathrm{Q}_{8} \times \mathrm{E}_{2} s, s \geq 0$.

(c) $G$ has an abelian maximal subgroup $A$ of exponent $>2$ and an element $x \in G-A$ of order 4 which inverts each element in $A$. 
